\title{
Which Chessboards have a Closed Knight's Tour within the Rectangular Prism?
}

\author{
Joe DeMaio \\ Department of Mathematics and Statistics \\ Kennesaw State University \\ Kennesaw, Georgia, 30144, USA \\ jdemaio@kennesaw.edu
}

\author{
Bindia Mathew \\ Department of Mathematics and Statistics \\ Kennesaw State University \\ Kennesaw, Georgia, 30144, USA \\ bmathew@students . kennesaw . edu
}

Submitted: Aug 17, 2010; Accepted: Dec 6, 2010; Published: Jan 5, 2011

Mathematics Subject Classification: 05C45,00A08

\begin{abstract}
A closed knight's tour of a chessboard uses legal moves of the knight to visit every square exactly once and return to its starting position. In 1991 Schwenk completely classified the $m \times n$ rectangular chessboards that admit a closed knight's tour. In honor of the upcoming twentieth anniversary of the publication of Schwenk's paper, this article extends his result by classifying the $i \times j \times k$ rectangular prisms that admit a closed knight's tour.
\end{abstract}

\section{Introduction}

The closed knight's tour of a chessboard is a classic problem in mathematics. Can the knight use legal moves to visit every square on the board and return to its starting position? The two dimensional movement of the knight makes its tour an intriguing problem which is trivial for other chess pieces. Euler presents solutions for the $8 \times 8$ board in a 1759 paper [4]. Martin Gardner discusses the knight's tour on rectangular boards and other mathematical problems involving the knight in his October 1967 column in Scientific American [5]. Papers exist analyzing the closed knight's tour on variant chessboards such as the cylinder [12], the torus [13], the sphere [1], the exterior of the cube [9] and the interior of the cube [3]. Donald Knuth generalizes the study of the $\{1,2\}$-knight on a rectangular board to the $\{r, s\}$-leaper on a rectangular board [8]. Across the Board: The Mathematics of Chessboard Problems by John Watkins is an indispensable collection of knight's tour results as well as many other mathematically themed chessboard problems [11].

Generalizing away from the chessboard, the closed knight's tour is a subset of the well known problem of the existence of Hamiltonian cycles in graphs. Despite the prior 
appearance of a paper [7] by Thomas Kirkman posing the general question, this cycle's name originates from mathematician William Rowen Hamilton and his Icosian game of the late 1850's. Photographs of the actual game can be viewed at http: //puzzlemuseum . com/month/picm02/200207icosian.htm, as hosted by The Puzzle Museum. Hamilton's Icosian game challenged players to visit every city on the board exactly once and return home.

Many results about closed knight's tours for rectangular boards had appeared in the literature throughout the years but no complete characterization of the solution was known until 1991. It was then that Schwenk completely answered the question: Which rectangular chessboards have a closed knight's tour [10]?

Theorem 1 (Schwenk) An $m \times n$ chessboard with $m \leq n$ has a closed knight's tour unless one or more of the following three conditions hold:

(a) $m$ and $n$ are both odd;

(b) $m \in\{1,2,4\}$;

(c) $m=3$ and $n \in\{4,6,8\}$.

To honor the twentieth anniversary of Schwenk's Theorem, we extend the result to $i \times j \times k$ rectangular prisms for integers $i, j, k \geq 2$.

Theorem 2 An $i \times j \times k$ chessboard for integers $i, j, k \geq 2$ has a closed knight's tour unless, without loss of generality, one or more of the following three conditions hold:

(a) $i, j$ and $k$ are all odd;

(b) $i=j=2$;

(c) $i=2$ and $j=k=3$.

To begin, consider two views of a closed knight's tour on the $2 \times 5 \times 6$ board. When presenting a board for the first time, we will always display the slices as in Figure 1. Note that this three dimensional tour is not just a combination of two copies of a closed knight's tour of the $5 \times 6$ board.

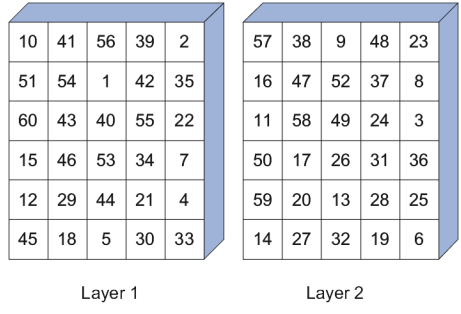

Figure 1: Slices of the $2 \times 5 \times 6$ board

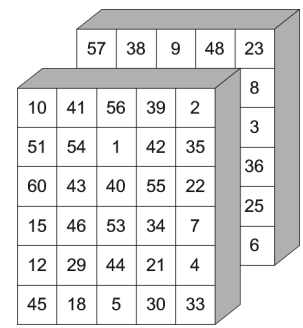

Figure 2: The 3 -D view of the $2 \times 5 \times 6$ board 
For the two-dimensional case the existence or non-existence of the $m \times n$ board clearly settles the question for the $n \times m$ board after a 90-degree rotation either clockwise or counterclockwise. The same holds true in three dimensions, although more options for rotations exist.

\section{Boards without Tours}

We first proceed by showing that the boards that satisfy at least one of the conditions of Theorem 2 do not contain a closed knight's tour. Parity conditions on $i, j$ and $k$ immediately dictate a necessary condition. A closed knight's tour does not exist on the $i \times j \times k$ rectangular prism for $i, j, k \equiv 1 \bmod 2$. The moves of the knight alternate color on the chessboard as shown in Figure 3 by the $a-b, c-d$ and $e-f$ moves. Thus, the knight's graph is bipartite. A closed knight's tour is an alternating cycle of black and white cells. Clearly, the number of white cells must equal the number of black cells. However, if $i, j$ and $k$ are all odd then the number of cells on the board is odd and the number of black cells cannot equal the number of white cells. Thus, no closed knight's tour exists on the $i \times j \times k$ chessboard when $i, j$ and $k$ are all odd.

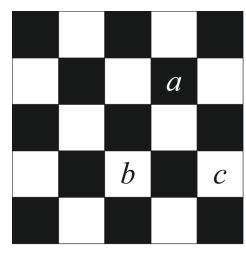

Layer 1

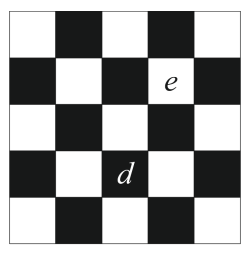

Layer 2

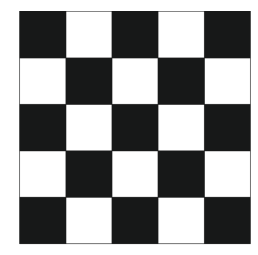

Layer 3

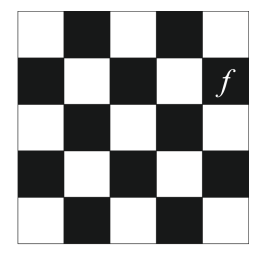

Layer 4

Figure 3: Knight moves on the $4 \times 5 \times 5$ board

It is a necessary condition that at least one of $i, j$ and $k$ be even. It is almost a sufficient condition as well. Almost, but not quite. A closed knight's tour does not exist on the $2 \times j \times 2$ board. The labeling of the cells in the $2 \times j \times 2$ board of Figure 4 shows that the knight's moves on the board are constrained. A knight can only move to and from cells of the same label. The knight's graph on the $2 \times j \times 2$ board is a disconnected graph. For the $3 \times 3 \times 2$ board, isolated vertices exist in the knight's graph as shown by the shaded cells in Figure 5. Naturally, a Hamiltonian cycle cannot exist in a disconnected graph or one with isolated vertices. 


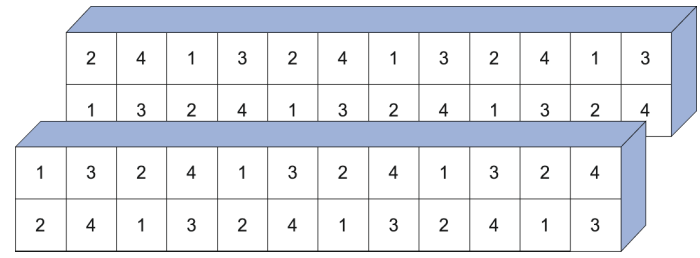

Figure 4: The $2 \times j \times 2$ rectangular prism

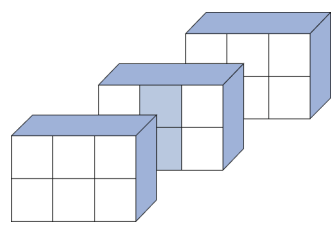

Figure 5: The $3 \times 3 \times 2$

Rectangular

Prism

\section{General Method to Create Tours}

We now prove the existence of a closed knight's tour for all other boards by constructing a tour for each board. Proof of the existence of a closed knight's tour for all other boards will be constructive and use the strong form of induction. As a gentle introduction to the reader we'll begin with examples of the three types of constructions we employ for the $i \times j \times k$ boards. We will use multiple copies of a closed knight's tour on a $2 \times 4 \times 4$ board to illustrate the process. Figure 6 shows the two layers of the $2 \times 4 \times 4$ board while Figure 7 illustrates the $2 \times 4 \times 4$ board in three dimensions.
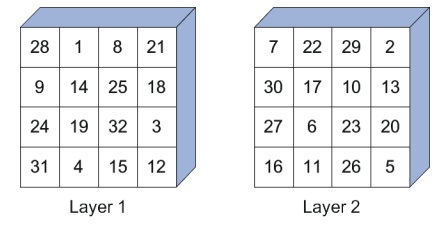

Figure 6: A $2 \times 4 \times 4$ closed knight's tour

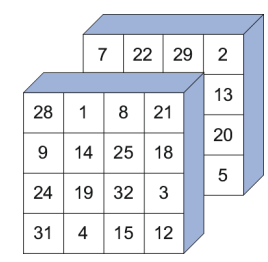

Figure 7:

The 3-D

view of the

$2 \times 4 \times 4$

board

The constructions in our proof begin with two closed knight's tours on two boards sharing at least two common parameters. We place the boards adjacent to each other to create a larger board. By selectively deleting key edges and creating new edges, we create a single closed knight's tour that traverses the new larger board. We use three methods to extend boards that share a common parameter: vertical stacking, horizontal stacking and front stacking. In vertical stacking, we place copies of the $2 \times 4 \times 4$ board on top of each other as shown in Figure 8 to create a $2 \times 4 \times 8$ board. We now want to combine the two disjoint closed knight's tours into one tour that tours every cell of the new $2 \times 4 \times 8$ 
board exactly once. We achieve this by deleting the $3-4$ edge on the top $2 \times 4 \times 4$ board and the $8-9$ edge on the bottom $2 \times 4 \times 4$ board and then creating the $3-8$ and $4-9$ edges to connect the previously disjoint tours into one single closed knight's tour for the $2 \times 4 \times 8$ board.

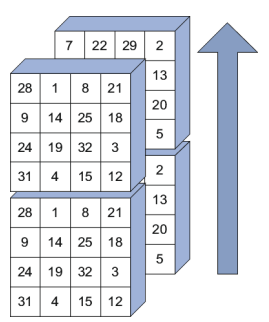

Figure 8:

Vertical stacking of two copies of the $2 \times 4 \times 4$

board

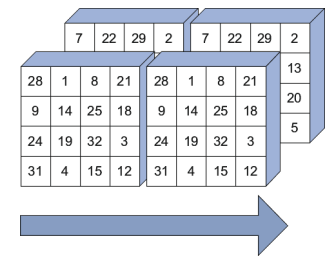

Figure 9:

Horizontal stacking of two copies of the $2 \times 4 \times 4$ board

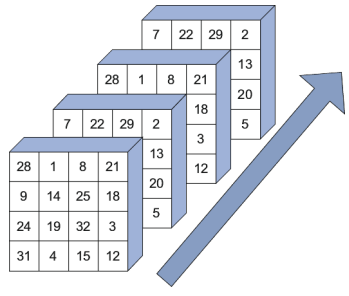

Figure 10: Front stacking of two copies of the $2 \times 4 \times 4$ board

Next we proceed with horizontal stacking of two copies of the $2 \times 4 \times 4$ board to create a $2 \times 8 \times 4$ board as illustrated in Figure 9. Delete the $25-26$ edge of the left $2 \times 4 \times 4$ board and the $27-28$ edge of the right $2 \times 4 \times 4$ board. Now create the $25-28$ and $26-27$ edges.

Finally we front stack two copies of the $2 \times 4 \times 4$ board to create a $4 \times 4 \times 4$ board. Delete the $10-11$ edge of the front $2 \times 4 \times 4$ board and the $14-15$ edge of the back $2 \times 4 \times 4$ board. Now create the $10-15$ and $11-14$ edges.

Using strong induction and the $2 \times 4 \times 4$ board, it is possible to construct a closed knight's tour on the $i \times j \times k$ for $i \equiv 0 \bmod 2$ and $j, k \equiv 0 \bmod 4$. If only a closed knight's tour existed for the $2 \times 2 \times 2$ board, our task would be much simpler! This clean and relatively simple example using only the $2 \times 4 \times 4$ board encompasses the range of techniques that constitute our entire proof. Many different boards will be required for the complete proof for all the possible values of $i, j$ and $k$.

\section{Boards with Tours}

Using the technique demonstrated in the previous section, we need to show how to construct a tour for all other boards not forbidden by Theorem 2. This forthcoming process will be very detailed but conceptually no harder than what we have already done. Since not all three values for $i, j$ and $k$ can be odd we will without loss of generality assume that $i \equiv 0 \bmod 2$. We will continue to utilize the $2 \times 4 \times 4$ board and introduce new boards as needed. We have already created a tour for any $i \times j \times k$ board where $i \equiv 0 \bmod 2$ and $j, k \equiv 0 \bmod 4$. Now we construct a tour for the other three values of $k \bmod 4$ while 
fixing $i \equiv 0 \bmod 2$ and $j \equiv 0 \bmod 4$. Figure 11 presents the $2 \times 4 \times 5$ base board for constructing the tours where $k \equiv 1 \bmod 4$. From here on out, we provide the details of the three stacking methods via tables to conserve space.

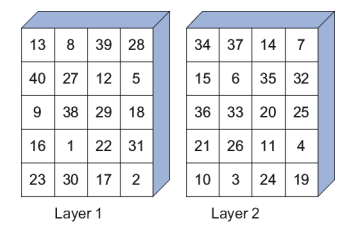

Figure 11: A $2 \times 4 \times 5$ closed knight's tour

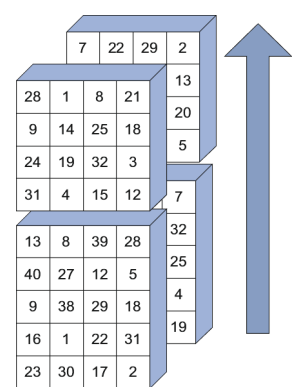

Figure 12:

Vertical stacking of the $2 \times 4 \times 5$ board below the $2 \times 4 \times 4$ board of Figure 6

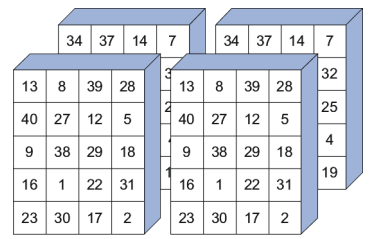

Figure 13:

Horizontal stacking of two copies of the $2 \times 4 \times 5$ board

\begin{tabular}{|l|l|l|}
\hline & Delete edges & Create edges \\
\hline Vertical & $3-4$ top board, $39-40$ bottom board & $3-39,4-40$ \\
\hline Horizontal & $31-32$ left board, $37-38$ right board & $31-38,32-37$ \\
\hline Front & $10-11$ front board, $16-17$ back board & $10-17,11-16$ \\
\hline
\end{tabular}

For $k \equiv 2 \bmod 4$, Figure 14 provides the $2 \times 4 \times 6$ base board.

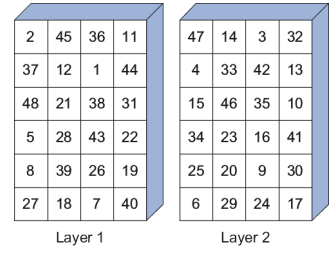

Figure 14: A

$2 \times 4 \times 6$ closed knight's tour

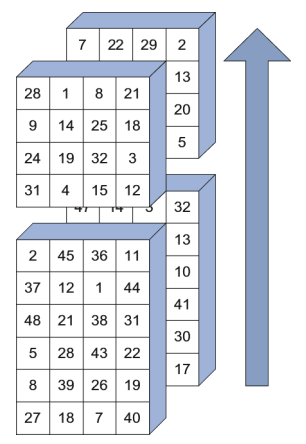

Figure 15:

Vertical stacking of the $2 \times 4 \times 6$ board below the $2 \times 4 \times 4$ board of Figure 6

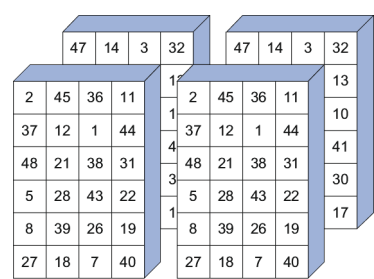

Figure 16:

Horizontal stacking of two copies of the $2 \times 4 \times 6$ board 


\begin{tabular}{|l|l|l|}
\hline & Delete edges & Create edges \\
\hline Vertical & $3-4$ top board, $36-37$ bottom board & $3-36,4-37$ \\
\hline Horizontal & $30-31$ left board, $28-29$ right board & $28-31,29-30$ \\
\hline Front & $29-30$ front board, $39-40$ back board & $29-40,30-39$ \\
\hline
\end{tabular}

And finally, a $2 \times 4 \times 3$ base board for $k \equiv 3 \bmod 4$.

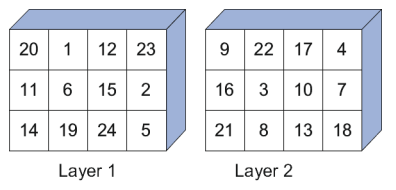

Figure 17: A $2 \times 4 \times 3$ closed knight's tour

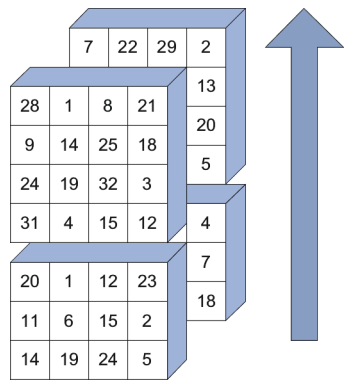

Figure 18: Vertical stacking of the $2 \times 4 \times 3$ board below the $2 \times 4 \times 4$ board of Figure 6

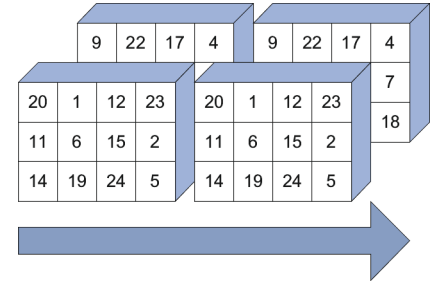

Figure 19: Horizontal stacking of two copies of the $2 \times 4 \times 3$ board

\begin{tabular}{|l|l|l|}
\hline & Delete edges & Create edges \\
\hline Vertical & $3-4$ top board, $11-12$ bottom board & $3-12,4-11$ \\
\hline Horizontal & $4-5$ left board, $20-21$ right board & $4-21,5-20$ \\
\hline Front & $7-8$ front board, $5-6$ back board & $5-8,6-7$ \\
\hline
\end{tabular}

At this point we have constructed a closed knight's tour for any $i \times j \times k$ board for $i \equiv 0 \bmod 2, j \equiv 0 \bmod 4$ and $k>2$. Next we cover the case of $i \equiv 0 \bmod 2$, $j \equiv 1 \bmod 4$ and $k>2$. We will extend these boards in three dimensions using our usual techniques. Previously we've used the $2 \times 4 \times 4$ board of Figure 6 in the inductive step. Now we use the $2 \times 4 \times 5$ board from Figure 11 in the inductive step. We begin with a $2 \times 5 \times 5$ base board. 


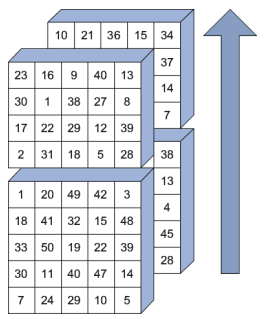

Figure 21:

Vertical stacking of the $2 \times 5 \times 5$ board below the $2 \times 5 \times 4$ board of Figure 11

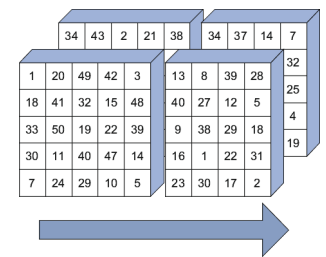

Figure 22:

Horizontal stacking of the $2 \times 5 \times 5$ board and the $2 \times 5 \times 4$ board of Figure 11

\begin{tabular}{|l|l|l|}
\hline & Delete edges & Create edges \\
\hline Vertical & $4-5$ top board, $20-21$ bottom board & $4-21,5-20$ \\
\hline Horizontal & $47-48$ left board, $8-9$ right board & $8-48,9-47$ \\
\hline Front & $43-44$ front board, $49-50$ back board & $43-50,44-49$ \\
\hline
\end{tabular}

Next, we create the $2 \times 5 \times 6$ base board and its extensions.

\begin{tabular}{|c|c|c|c|c|}
\hline 1 & 54 & 59 & 18 & 3 \\
\hline 60 & 19 & 10 & 43 & 56 \\
\hline 51 & 42 & 55 & 48 & 39 \\
\hline 12 & 47 & 44 & 23 & 8 \\
\hline 15 & 52 & 33 & 40 & 5 \\
\hline 34 & 25 & 6 & 45 & 22 \\
\hline 34 & \multicolumn{3}{|c|}{ Layer 1 11} \\
\hline 11 & 36 & 57 & 20 & 9 \\
\hline 16 & 53 & 50 & 37 & 4 \\
\hline 35 & 26 & 29 & 46 & 21 \\
\hline 32 & 41 & 14 & 27 & 30 \\
\hline 13 & 28 & 31 & 24 & 7 \\
\hline
\end{tabular}

Figure 23: A $2 \times 5 \times 6$ closed knight's tour

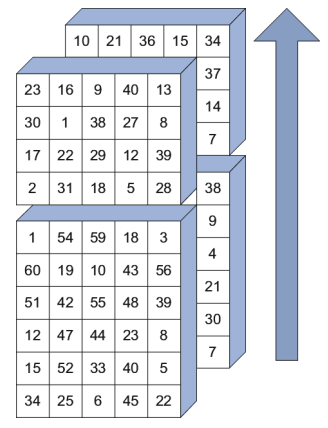

Figure 24:

Vertical stacking of the $2 \times 5 \times 6$ board below the rotated $2 \times 5 \times 4$ board of

Figure 11

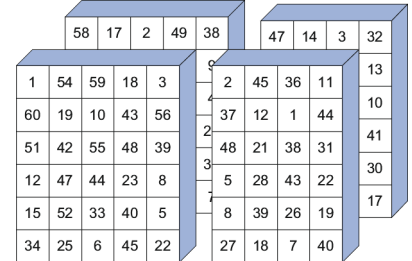

Figure 25: Horizontal stacking of the

$2 \times 5 \times 6$ board and the $2 \times 4 \times 6$ board of Figure 14 


\begin{tabular}{|l|l|l|}
\hline & Delete edges & Create edges \\
\hline Vertical & $11-12$ top board, $2-3$ bottom board & $2-11,3-12$ \\
\hline Horizontal & $37-38$ left board, $33-34$ right board & $33-38,34-37$ \\
\hline Front & $57-58$ front board, $59-60$ back board & $57-60,58-59$ \\
\hline
\end{tabular}

Once more for the $2 \times 5 \times 3$ board.

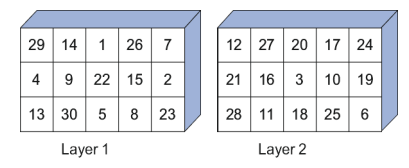

Figure 26: A

$2 \times 5 \times 3$ closed

knight's tour

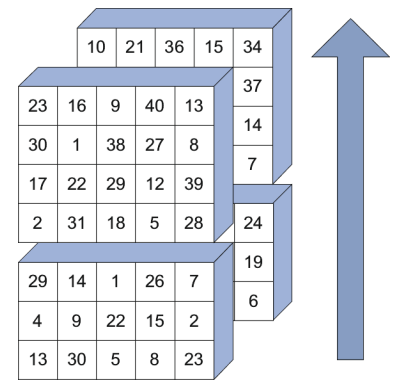

Figure 27: Vertical stacking of the $2 \times 5 \times 3$ board below the rotated $2 \times 4 \times 5$ board of

Figure 11

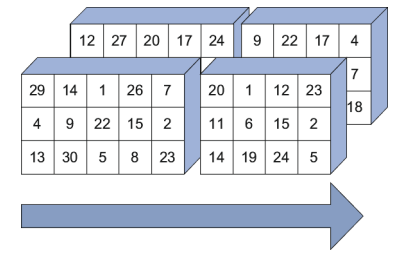

Figure 28:

Horizontal stacking of the $2 \times 5 \times 3$

board and the $2 \times 4 \times 3$ board of

Figure 17

\begin{tabular}{|l|l|l|}
\hline & Delete edges & Create edges \\
\hline Vertical & $17-18$ top board, $14-15$ bottom board & $14-17,15-18$ \\
\hline Horizontal & $23-24$ left board, $20-21$ right board & $20-23,21-24$ \\
\hline Front & $27-28$ front board, $29-30$ back board & $27-30,28-29$ \\
\hline
\end{tabular}

There is no need for a $2 \times 5 \times 4$ board for the case of $i \equiv 0 \bmod 2, j \equiv 1 \bmod 4$ and $k \equiv 0 \bmod 4$ as that case is covered by a rotation of the board created for the $i \equiv 0 \bmod 2, j \equiv 0 \bmod 4$ and $k \equiv 1 \bmod 4$ case.

Continuing with our strategy, we proceed to create a closed knight's tour on all $i \times j \times k$ boards for $i \equiv 0 \bmod 2, j \equiv 2 \bmod 4$ and $k>2$. We begin with a $2 \times 6 \times 6$ base board (since no $2 \times 2 \times k$ tour exists) and use the $2 \times 4 \times 6$ board of Figure 14 to extend. 

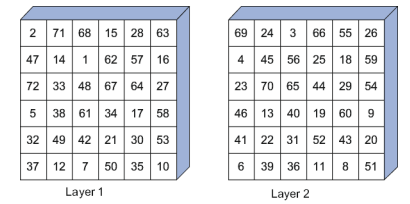

Figure 29: A $2 \times 6 \times 6$ closed knight's tour

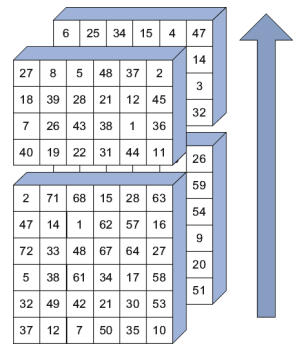

Figure 30:

Vertical stacking of the $2 \times 6 \times 6$ board below the rotated $2 \times 4 \times 6$ board of Figure 14

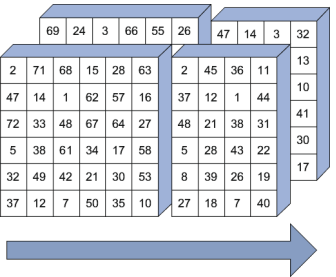

Figure 31:

Horizontal stacking of the $2 \times 6 \times 6$ board and the $2 \times 4 \times 6$ board of Figure 14

\begin{tabular}{|l|l|l|}
\hline & Delete edges & Create edges \\
\hline Vertical & $43-44$ top board, $15-16$ bottom board & $15-43,16-44$ \\
\hline Horizontal & $29-30$ left board, $4-5$ right board & $4-29,5-30$ \\
\hline Front & $22-23$ front board, $32-33$ back board & $22-33,23-32$ \\
\hline
\end{tabular}

Once again for the $2 \times 6 \times 3$ board.

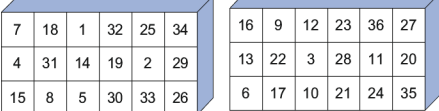

Layer 1

Layer 2

Figure 32: A $2 \times 6 \times 3$ closed knight's tour

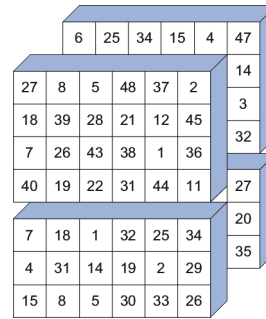

Figure 33: Vertical stacking of the $2 \times 6 \times 3$ board below the $2 \times 6 \times 4$ board of Figure 14

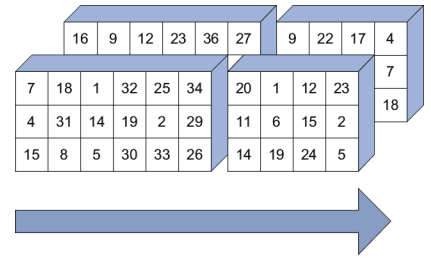

Figure 34: Horizontal stacking of the $2 \times 6 \times 3$ board and the $2 \times 4 \times 3$ board of Figure 17

\begin{tabular}{|l|l|l|}
\hline & Delete edges & Create edges \\
\hline Vertical & $9-10$ top board, $11-12$ bottom board & $9-12,10-11$ \\
\hline Horizontal & $26-27$ left board, $20-21$ right board & $20-26,21-27$ \\
\hline Front & $22-23$ front board, $18-19$ back board & $18-23,19-22$ \\
\hline
\end{tabular}


Now on to the $i \times j \times k$ boards for $i \equiv 0 \bmod 2, j \equiv 3 \bmod 4$ and $k>2$. The non-existence of a $2 \times 3 \times 3$ board forces us to use a $2 \times 7 \times 3$ closed knight's tour as a base case. To extend it in three dimensions, we vertically stack it with a $2 \times 7 \times 4$ board. This extension is a 90-degree rotation of the $2 \times 4 \times 7$ board which appeared in Figure 18 . For clarity, we renumber the cells of the board from Figure 18 with labels 1 through 56 to create the board in Figure 36.

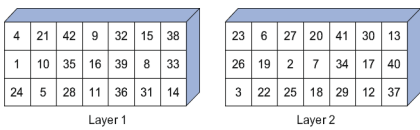

Figure 35: A $2 \times 7 \times 3$ closed knight's tour

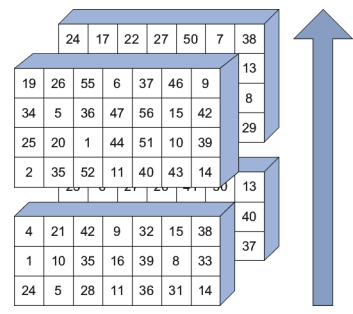

Figure 37: Vertical stacking of the $2 \times 7 \times 3$ board below the $2 \times 7 \times 4$ board of Figure 36

\begin{tabular}{|c|c|c|c|c|c|c|}
\hline 19 & 26 & 55 & 6 & 37 & 46 & 9 \\
\hline 34 & 5 & 36 & 47 & 56 & 15 & 42 \\
\hline 25 & 20 & 1 & 44 & 51 & 10 & 39 \\
\hline 2 & 35 & 52 & 11 & 40 & 43 & 14 \\
\hline
\end{tabular}

\begin{tabular}{|c|c|c|c|c|c|c|}
\hline 24 & 17 & 22 & 27 & 50 & 7 & 38 \\
\hline 3 & 32 & 49 & 16 & 41 & 28 & 13 \\
\hline 18 & 23 & 54 & 21 & 30 & 45 & 8 \\
\hline 33 & 4 & 31 & 48 & 53 & 12 & 29 \\
\hline
\end{tabular}

Figure 36: A $2 \times 7 \times 4$ closed knight's tour

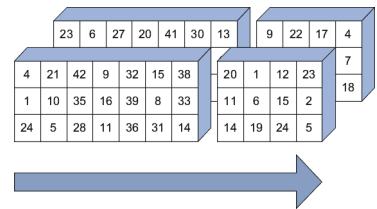

Figure 38:

Horizontal stacking

of the $2 \times 7 \times 3$

board and the

$2 \times 4 \times 3$ board of

Figure 17

\begin{tabular}{|l|l|l|}
\hline & Delete edges & Create edges \\
\hline Vertical & $20-21$ top board, $7-8$ bottom board & $7-20,8-21$ \\
\hline Horizontal & $13-14$ left board, $20-21$ right board & $13-21,14-20$ \\
\hline Front & $12-13$ front board, $14-15$ back board & $12-15,13-14$ \\
\hline
\end{tabular}

We have almost demonstrated how to create all $2 \times j \times k$ boards that permit a closed knight's tour according to Theorem 2. At first glance it seems that we have covered all permitted combinations of $i, j$ and $k$. However, the non-existence of the $2 \times 3 \times 3$ board prevented us from creating one particular case of boards; all $i \times 3 \times 3$ where $i \equiv 0 \bmod 2$. Constructing the $4 \times 3 \times 3$ and $6 \times 3 \times 3$ base boards and presenting the method to let $i$ assume any even value allows us to complete the proof of Theorem 2 . We can create all $i \times 3 \times 3$ boards where $i \equiv 0 \bmod 4$ with Figure 39 . 

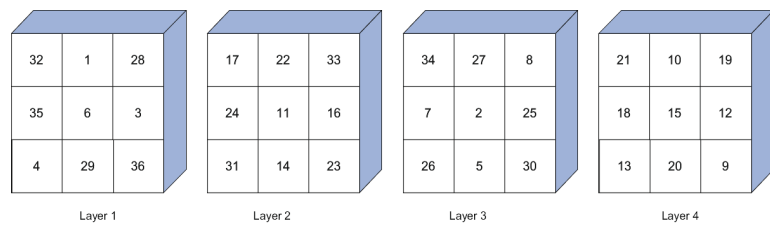

Figure 39: Slices of a $4 \times 3 \times 3$ closed

knight's tour

\begin{tabular}{|l|l|l|}
\hline & Delete edges & Create edges \\
\hline Front & $9-10$ front board, $28-29$ back board & $9-28,10-29$ \\
\hline
\end{tabular}

For $i \times 3 \times 3$ boards where $i \equiv 2 \bmod 4$ we begin with Figure 40 and continually front stack Figure 39.

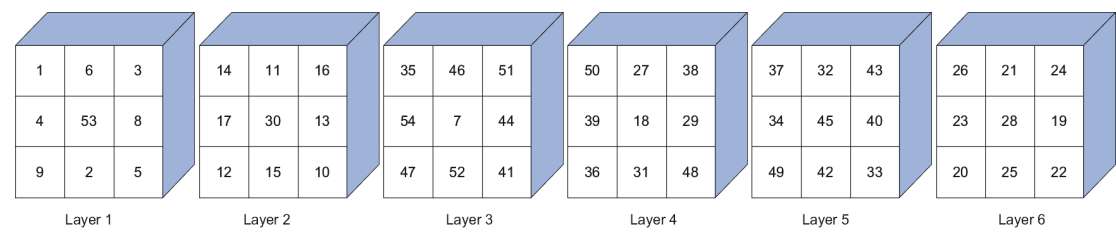

Figure 40: Slices of a $6 \times 3 \times 3$ closed knight's tour

\begin{tabular}{|l|l|l|}
\hline & Delete edges & Create edges \\
\hline Front & $21-22$ front board, $28-29$ back board & $21-29,22-28$ \\
\hline
\end{tabular}

\section{Future Work}

One could pursue an increase in the number of dimensions with a $1-2$ knight by searching for closed knight's tours in four dimensions. Once we move to $n \geq 4$ dimensions we lose the ability to easily visualize the geometry of a closed knight's tour. One approach would use vectors of length $n$ to represent the cells of the board. A legal move of the knight from one square to another square would change two of the vector coordinates from the initial square. One coordinate would change by \pm 1 and the other by \pm 2 . A very ambitious project would be to find a general classification for the existence of a closed knight's tour in the $n$ dimensional cube where Theorems 1 and 2 in this paper are just the specific cases for $n=2,3$.

Another option explores the nature of the move of the knight [9]. On the two dimensional board, the knight's move incorporates both directions in the $x-y$ plane. One could argue that the move of the knight on the three dimensional board should incorporate all three directions in the $x-y-z$ plane. How should the knight move in the three dimensional board? Perhaps, the obvious variant piece is a $1-2-3$ knight. Unfortunately, the move of the $1-2-3$ knight in the three-dimensional board is not bipartite. Such a move 
would leave the knight locked into one color like the bishop. See [2] for an example of a tour of the cube of side $n=6$ with two $1-2-3$ knights, one for the black cells and one for the white cells.

Instead of a linear change in the number of cells the knight moves, let's consider an exponential change and use a $1-2-4$ knight in a three dimensional board. This move has an advantage over the $1-2-3$ knight since the graph of the $1-2-4$ knight is bipartite. As a teaser, in Figure 41, we leave you with a $1-2-4$ closed knight's tour of the cube of side 8 , the smallest cube that admits such a tour.

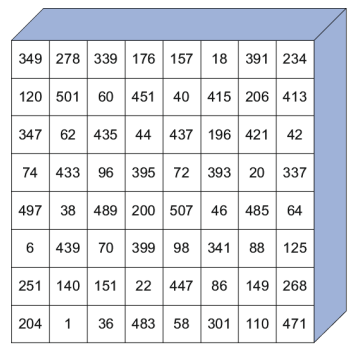

Layer 1

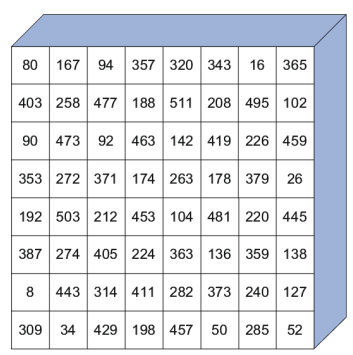

Layer 4

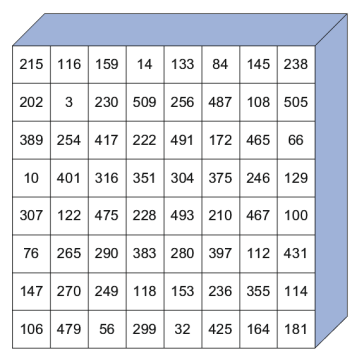

Layer 7

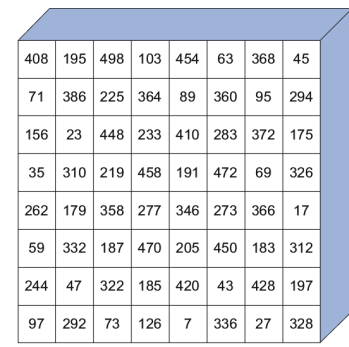

Layer 2

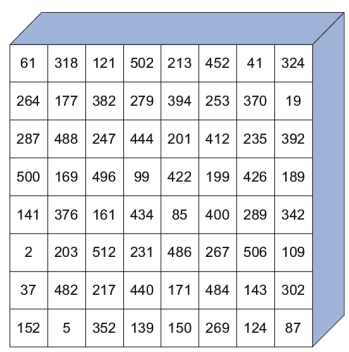

Layer 5

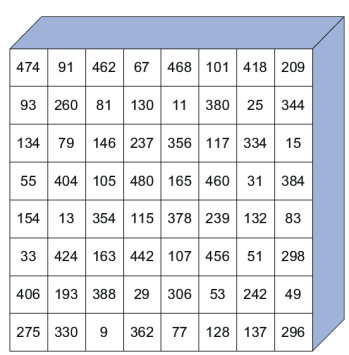

Layer 8

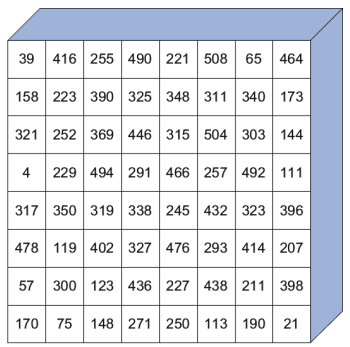

Layer 3

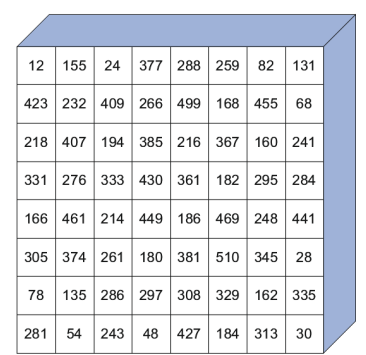

Layer 6

Figure 41: A $1-2-4$ closed knight's tour of the cube of side 8

\section{Acknowledgements}

The authors would like to thank Michael Schornak for his contributions to this project. Michael wrote a program that finds closed knight's tours in three dimensional boards. When constructing base boards for inductive purposes, Michael's program proved to be a 
priceless resource. We would also like to thank the Mentor-Protege program at Kennesaw State University for supporting this research. We appreciate the thoughtful and insightful comments by the anonymous referee which improved the clarity of the presentation of our work. And finally, we thank Dr. Philippe Laval for his help with the technical details of LTEX and Scientific Workplace.

\section{References}

[1] G. Cairns, Pillow Chess, Mathematics Magazine, 75, no. 3. (June, 2002), 173-186.

[2] J. DeMaio, Closed Monochromatic Bishops' Tour, Journal of Recreational Mathematics, 34, no. 3, pp. 196-203

[3] J. DeMaio, Which Chessboards have a Closed Knight's Tour within the Cube?, The Electronic Journal of Combinatorics, 14, (2007) R32.

[4] L. Euler, Solutio d'une Question Curieuse qui ne Peroit Soumise a Aucune Analyse, Mem. Acad. Sci. Berlin 15 (1759), 310-337.

[5] M. Gardner, Problems that are built on the knight's move in chess, Scientific American, 217 (1967) no. 4, 128-132.

[6] R. J. Gould, Hamiltonian Graphs, Handbook of Graph Theory, CRC Press, Boca Raton, (2004), 261-278.

[7] T. P. Kirkman, On the Representation of Polyhedra, Philosophical Transactions of the Royal Society (London) 146 (1856), 413-418

[8] D. E. Knuth, Leaper Graphs, Math. Gazette 78 (1994), 274-297.

[9] Y. Qing and J.J. Watkins, Knight's Tours for Cubes and Boxes, Congressus Numerantium 181 (2006) 41-48.

[10] A. J. Schwenk, Which Rectangular Chessboards have a Knight's Tour? Mathematics Magazine 64:5 (December 1991) 325-332.

[11] J. J. Watkins, Across the Board: The Mathematics of Chessboard Problems, Princeton University Press, Princeton, 2004.

[12] J. J. Watkins, Knight's tours on cylinders and other surfaces, Congr. Numer. 143 (2000), 117-127.

[13] J. J. Watkins, Knight's tours on a torus, Mathematics Magazine, 70:3 (1997), 175184.

[14] D. B. West, Introduction to Graph Theory, Second Edition, Prentice Hall, Upper Saddle River, 2001. 